\title{
Bibliographische Notizen
}

Anca Gheaus, PhD, Assistant Professor für Philosophie an der Central European University Wien.

Forschungsschwerpunkte: Angewandte Ethik, politische Philosophie, Theorie der Gerechtigkeit, Kinder, Kindheit.

Constanze Giese, Prof. Dr. theol., examinierte Krankenschwester, Professorin für Ethik und Anthropologie in der Pflege, Katholische Stiftungshochschule München.

Forschungsschwerpunkte: Ethik in Pflege und Pflegebildung, Professionsentwicklung und sozialethische Fragen der Pflege.

Grunwald, Armin, Prof. Dr. rer nat., Professor für Technikphilosophie am Institut für Philosophie des Karlsruher Instituts für Technologie und Leiter des Büros für Technikfolgen-Abschätzung beim Deutschen Bundestag.

Forschungsschwerpunkte: Theorie der Technikfolgenabschätzung, Ethik der Technik, Konzeption der Nachhaltigkeit, Digitale Transformation.

Andreas Heinz, Prof. Dr. med. Dr. phil., Direktor der Klinik für Psychiatrie und Psychotherapie der Charité - Universitätsmedizin Berlin.

Forschungsschwerpunkte: Lernmechanismen bei psychischen Störungen, Computergestützte Modelle psychotischer Erfahrungen, Interkulturelle Psychiatrie und Psychotherapie, Migration und psychische Gesundheit.

Christoph Kehl, Dr. phil., Wissenschaftlicher Mitarbeiter des Büros für Technikfolgen-Abschätzung beim Deutschen Bundestag (TAB).

Forschungsschwerpunkte: Bio- und Neurotechnologien, Robotik/KI sowie Umwelt und Nachhaltigkeit

Heribert Kentenich, Prof. Dr. med., Frauenarzt, Mitglied von Arbeitsgruppen der Bundesärztekammer und der Nationalen Akademie der Wissenschaften Leopoldina.

Forschungsschwerpunkte: Reproduktionsmedizin, Psychotherapie.

Krüger, Hans-Peter, Prof. Dr. phil., em. Professor für Politische Philosophie und Philosophische Anthropologie am Institut für Philosophie der Universität Potsdam.

Forschungsschwerpunkte: Philosophische Anthropologie, Philosophischer Pragmatismus, politische Philosophie (insb. Verhältnis des Privaten und Öffentlichen), Sozialphilosophie (insb. gesellschaftliche Kommunikation, öffentliche Lernprozesse).

Olivia Mitscherlich-Schönherr, Dr. phil. habil., Dozentin für Philosophische Anthropologie mit Schwerpunkt auf Grenzfragen des Lebens an der Hochschule für Philosophie München. Forschungsschwerpunkte: Philosophische Anthropologie, Lebenskunst, Philosophie der Liebe, Sympathieethik, philosophische Bioethik, Biopolitik, Philosophie der Philosophie. 
Jos de Mul, Prof. Dr. phil., Professor für Philosophische Anthropologie an der Erasmus School of Philosophy der Erasmus University Rotterdam.

Forschungsschwerpunkte: philosophische Anthropologie, Philosophie der Biologie, Technikphilosophie, Ästhetik und Geschichte der deutschen Philosophie des 19. und 20. Jahrhunderts.

Oliver Müller, Prof. Dr. phil., Professor für Philosophie am Philosophischen Seminar der Albert-Ludwigs-Universität Freiburg.

Forschungsschwerpunkte: Philosophie der Technik, philosophische Anthropologie, Naturphilosophie und Ethik.

Tobias Müller, Dr. phil. habil., Vertretungsprofessor für Religionsphilosophie an der GoetheUniversität Frankfurt.

Forschungsschwerpunkte: Religionsphilosophie, Philosophie des Geistes, Natur- und Technikphilosophie.

Petra Schaper Rinkel, Prof. Dr. rer pol., Professorin für Wissenschafts- und Technikforschung des digitalen Wandels und Vizerektorin für Digitalisierung an der Universität Graz. Forschungsschwerpunkte: Digitalisierung, Foresight, Innovationsforschung, Governance von Zukunftstechnologien und Politische Theorie der Technik.

Johannes F.M. Schick, Dr. phil., Habilitand an der a.r.t.e.s. Graduate School for the Humanities der Universität zu Köln.

Forschungsschwerpunkte: Technikphilosophie, französische Philosophie des 20. Jahrhunderts, philosophische Anthropologie, das Verhältnis von Philosophie zu Ethnologie und die Philosophie der Emotionen.

Christina Schües, Prof. Dr. phil., Professorin für Philosophie am Institut für Medizingeschichte und Wissenschaftsforschung der Universität zu Lübeck, und apl. Prof. am Institut für Philosophie und Kunstwissenschaft der Leuphana Universität, Lüneburg.

Forschungsschwerpunkte: Conditio humana und mitmenschliche Beziehungsverhältnisse, Macht der Zeit, Phänomenologie, Anthropo-Technologien, Friedenstheorien, Sozial- und Medizinphilosophie.

Mark Schweda, Prof. Dr. phil., Professor für Ethik in der Medizin, Carl von Ossietzky Universität Oldenburg.

Forschungsschwerpunkte: Altern, Lebensverlauf und menschliche Zeitlichkeit sowie Technik und Digitalisierung in Medizin und Gesundheitswesen.

Assina Seitz, B.Sc. in Psychologie, B.A. in Journalismus, studentische Hilfskraft an der Klinik für Psychiatrie und Psychotherapie der Charité Berlin.

Tobias Sitter, MA in Philosophie, Student der Medizin an der TU München und der Universität Padua.

Forschungsschwerpunkte: philosophische Anthropologie, Subjektphilosophie, Naturphilosophie, Wissenschaftsphilosophie, Medizinethik. 
Björn Sydow, Dr. phil., Lehrkraft für besondere Aufgaben am Institut für Philosophie der JustusLiebig-Universität Gießen.

Forschungsschwerpunkte: Zusammenhang von praktischer Vernunft und moralischen Pflichten, Angewandte Ethik. 
